\title{
Anabases
}

ANABASES Traditions et réceptions de l'Antiquité

$7 \mid 2008$

Varia

\section{Harry Potter en latin}

\section{Jacques Elfassi}

\section{OpenEdition}

Journals

Édition électronique

URL : http://journals.openedition.org/anabases/2537

DOI : 10.4000/anabases.2537

ISSN : 2256-9421

\section{Éditeur}

E.R.A.S.M.E.

\section{Édition imprimée}

Date de publication : 1 mars 2008

Pagination : 231-237

ISSN : 1774-4296

\section{Référence électronique}

Jacques Elfassi, « Harry Potter en latin », Anabases [En ligne], 7 | 2008, mis en ligne le 01 décembre

2011, consulté le 21 octobre 2019. URL : http://journals.openedition.org/anabases/2537 ; DOI

10.4000/anabases.2537 
Anabases 7 (2008), p. 231-237.

\section{Harry Potter en latin}

JACQUES ELFASSI

EN 2003 ET EN 2007 OnT ÉTÉ PUBLIÉES les traductions latines des deux premiers tomes de Harry Potter de Joanne Kathleen Rowling: Harry Potter à l'école des sorciers et Harry Potter et la chambre des secrets ${ }^{1}$.

Sans doute ne faut-il pas accorder à ces traductions une importance disproportionnée. Ce ne sont certainement pas elles qui ont créé le succès de Harry Potter, elles ne font que l'accompagner ; et bien qu'on ne puisse pas les réduire à cette seule caractéristique, ce sont aussi des produits " marketing ", faisant partie de la stratégie commerciale d'un éditeur, Bloomsbury, qui est fier de pouvoir annoncer que la série des Harry Potter a déja été traduite dans plus de cinquante langues, " y compris " (" also ») des langues aussi rares et exotiques que le grec ancien, le latin, l'irlandais et le gallois ${ }^{2}$. Toutefois, de telles traductions ne constituent pas un phénomène isolé : d'autres ouvres de fiction ont récemment été traduites en latin. En se limitant aux vingt dernières années et en excluant délibérément la littérature enfantine (afin de prouver que ce phénomène ne concerne pas seulement des ouvrages pour la jeunesse comme Winnie the Pooh ou Astérix), il est possible de citer, parmi les romans traduits en latin, La famille de Pascual Duarte de Camilo José Cela (1990), Lornière de Hermann Hesse (1994), Don Quichotte de Miguel de Cervantès (1998), La nuit de Lisbonne d'Erich Maria Remarque (2002) ou Le parfum de Patrick Süskind (2004) ${ }^{3}$.

1 J.K. Rowling, Harrius Potter et Philosophi Lapis, Londres, 2003, et Harrius Potter et Camera Secretorum, Londres, 2007 ; les deux traductions sont dues à Peter Needham.

2 "The Harry Potter books are translated into over 50 languages " (rabat de la jaquette de Harrius Potter et Philosophi Lapis). Le verso de la page de "faux-titre ", dans la traduction en grec ancien (parue en 2004), précise : "Also available... Ancient Greek, Latin, Irish and Welsh editions ".

3 C.J. Cela, De Familia Pascual Duarte, Madrid, 1990 (traductrice : Bárbara Pastor de Arozena) ; H. Hesse, Sub Rota, Sarrebruck, 1994 (Sigrid C. Albert) ; M. DE CervanTES, Dominus Quixotus a Manica, Alcalá de Henares, 1998 (Antonio Peral Torres); E.M. RemARQUe, De nocte Olisiponensi, Sarrebruck, 2002 (Sigrid Albert) ; P. SüsKIND, Fragrantia, Bruxelles, 2004 (Nikolaus Groß). Je cite plusieurs de ces titres de seconde main : je n'ai consulté que les traductions de Don Quichotte et du Parfum. 
Les traductions latines récentes ont une spécificité par rapport à celles de l'époque moderne (du XVIe au XVIII siècle) : ces dernières permirent à certains lecteurs, notamment dans le monde germanophone, d'accéder à certains livres qu'ils n'auraient peut-être pas lus autrement, contribuant ainsi à la diffusion de certaines œuvres et de certaines idées 4 . A présent, en revanche, la connaissance du latin est tellement limitée que ce rôle n'existe plus. Comme l'écrit F. Waquet ${ }^{5}$, " aujourd'hui les traductions latines d'œuvres de fiction, telles que Pinocchio, Bonjour tristesse ou Astérix le Gaulois, sanctionnent plus un succès établi qu'elles ne le favorisent - qui est donc capable de les lire ? " Mais alors, comment expliquer qu'encore aujourd'hui on en produise et on en publie ? À quoi servent-elles?

La première explication qui vient à l'esprit est d'ordre pédagogique : ces livres sont censés faciliter l'apprentissage du latin en distrayant les élèves et en diminuant à leurs yeux le caractère lointain que peut avoir cette langue. C'est probablement ce qui explique la présence de ces traductions dans divers manuels de latin : celle de Pinocchio par Enrico Maffacini est incluse dans un manuel de $1962^{6}$, celle de La chèvre de Monsieur Seguin d'Alphonse Daudet dans un manuel de $1967^{7}$; plusieurs vignettes tirées des albums d'Astérix en latin sont reproduites dans des manuels plus récents ${ }^{8}$. Les deux volumes de Harrius Potter affichent aussi explicitement leur dessein pédagogique : le texte sur le rabat de la jaquette précise que ces deux livres "seront intéressants pour tous ceux qui étudient la langue (latine), que ce soit à l'école ou à un plus haut niveau " (" will be of interest to all those who are sudying the language, whether at school or at a higher level »). Il est notable d'ailleurs que le seul article (à ma connaissance) qui ait été consacré jusqu’à présent à la traduction latine de Harry Potter ait été publié dans une revue de didactique 9 .

4 Voir F. WaQUeT, Le latin ou l'empire d'un signe. XVIe-XXe siècle, Paris, 1998, p. 107-109; P. Burke, "Translations into Latin in early modern Europe", in P. Burke et R.P. Hsia (éd.), Cultural Translation in Early Modern Europe, Cambridge, 2007, p. 71.

Le latin [n. 4], p. 109.

6 C. Collodi, Pinoculus, Florence, 1951, reproduit par J.-A. de Foucault, Pratique du latin : classe de quatrième, Paris, Éditions J. de Gigord (collection M.-L. Podvin), 1965, p. 8, 24, 39, etc. (texte présenté sous forme de "feuilleton ", en 18 extraits).

M. Lefaure, "La chèvre de Monsieur Seguin", Vita Latina 1 (juin 1957), p. 17-21, reproduite par G. Griffe, Latin : classe de quatrième, Paris, Bordas (collection G. Bloch), 1967, p. $99-102$.

J. Gason et A. Lambert, Linvitation au latin. $4^{e}$ et 3e, Paris, 1988 et 1996, Paris, Magnard, passim (dans le manuel de $4^{e}$, p. 8, 14, etc., dans celui de $3{ }^{e}$, p. 38, 44, etc.). Les mêmes auteurs empruntent aussi à la traduction du Petit Prince (manuel de $4^{e}$, p. 20 et 26).

9 A. LOZAR, "Num legendus est ille liber cui titulus est 'Harrius Potter et philosophi lapis'? Einige Anmerkungem zur lateinischen Übersetzung von 'Harry Potter und der Stein der Weisen'”, Pegasus-Onlinezeitschrift. Wissensachftliches Periodikum zu Didaktik und Methodik der Fächer Latein und Griechisch 4/2 (2004), p. $46-49$ (http ://www.pegasusonlinezeitschrift.de/agora_2_2004_lozar_potter.html) ; je ne partage d'ailleurs pas les conclusions de cet article, injustement sévère envers la traduction de P. Needham. C'est aussi dans une perspective pédagogique que j'ai moi-même écrit une première ébauche de ce travail : "Harry Potter en latin", Kallirrhoé 17 (avril 2004), p. 34-35 (Kallirrhoé est le bulletin de "Kallirrhoé", association des étudiants de Lettres Classiques de l'Université de Metz). 
Il existe toutefois une autre raison, peut-être plus importante, qui explique la production et le succès (certes très relatif) de ces traductions : c'est leur aspect ludique. Aujourd'hui, alors que le latin est une langue de moins en moins étudiée et comprise, être capable de traduire un texte en latin et de lire cette traduction constitue un véritable jeu érudit ${ }^{10}$. Faire passer un texte de l'anglais au latin alors que le nombre de lecteurs latinophones est infiniment plus faible que celui de lecteurs anglophones et que Harry Potter décrit un monde bien plus éloigné de la Rome antique que de l'Angleterre actuelle présente un caractère canularesque. En ce cas, d'ailleurs, le latin ne joue pas seulement le rôle de langue canularesque, mais aussi de code secret réservé à des initiés : quand on lit ce genre de traduction, on se surprend à éprouver une certaine fierté de faire partie des happy few capables de comprendre la plaisanterie ${ }^{11}$.

Cependant l'humour est souvent plus profond lorsqu'il est prononcé sur un ton sérieux : le paradoxe d'une traduction latine est d'autant mieux mis en valeur que le latin est impeccable et le plus fidèle possible à l'original. C'est dans cette logique que je me propose, dans cet article, d'aborder la traduction de Harry Potter par Peter Needham : quelles difficultés a-t-il dû résoudre pour essayer d'être fidèle à son modèle ${ }^{12}$ ?

Sans être traductologue, on peut admettre comme principe général qu'une traduction, normalement, est destinée à rendre un texte compréhensible à des gens qui, parce qu'ils n'en connaissent pas la langue, ne peuvent pas le comprendre sans traduction, ou du moins, s'ils n'en connaissent qu'imparfaitement la langue, ne peuvent pas bien le comprendre. Par exemple, quand on traduit un texte anglais en français, c'est pour le faire comprendre, ou mieux le faire comprendre, à des francophones qui ne comprennent pas, ou ne comprennent pas bien l'anglais. Traduire, donc, c'est rapprocher un texte de ses lecteurs. Le cas des traductions latines récentes présente à cet égard un schéma atypique et paradoxal (y compris par rapport aux traductions latines du XVI e ou du XVII siècle) : en effet, la langue d'arrivée (le latin) est encore plus éloignée de la langue du lecteur (le français, par exemple) que la langue de départ (l'anglais, dans le cas de Harry Potter). Cette distance n'est d'ailleurs pas seulement linguistique, mais aussi culturelle : le lecteur d'aujourd'hui (francophone ou pas) est plus proche du monde de Harry Potter (l'Angleterre actuelle) que de celui de Harrius Potter (le monde romain antique auquel est géné-

10 "A learned game ", comme l'écrit L.G. Kelly, "Latin Tradition", in M. Baker et K. Malmkjaer (éd.), Routledge Encyclopedia of Translation Studies, Londres-New York, 1998, p. 503. Formule presque identique (" a form of learned playfulness ") chez P. BuRKE, "Culture of translation in early modern Europe", in Burke et Hsia, Cultural Translation [n. 4], p. 19.

11 Sur l'usage du latin comme langue de canular et comme " code secret ", voir à nouveau Waquet, Le latin [n. 4], p. 139.

12 Bien que leur perspective soit un peu différente de la mienne, je signale deux articles qui se proposent aussi d'analyser des traductions latines récentes d'un point de vue philologique : J.Ma. CASASAYAS, "Iam erat hora diluculi. Cuando apareció otro Dominus Quixotus", in I. Lozano-Renieblas et J.C. Mercado (éd.), Silva. Studia philologica in honorem Isaías Lerner, Madrid, 2001, p. 147-152 ; et M. JACQMAIN, "Duttilità e perennità de la lingua latina : Pinoculus", Chroniques Italiennes, série web 7 (2005), 11 pages.

(http ://www.univ-paris3.fr/recherche/chroniquesitaliennes/PDF/Web7/Jacqmain.pdf). 
ralement associée la langue latine). Les traductions latines récentes sont par définition anachroniques.

Cet anachronisme est conscient et délibéré, car l'humour de ces traductions latines est précisément fondé sur l'effet de distanciation qui en résulte. Dans le texte original (en anglais) de Harry Potter, le latin est d'ailleurs aussi utilisé par l'auteur, J.K. Rowling, pour créer une distanciation par rapport au lecteur moderne, afin de suggérer un univers médiéval ou pseudo-médiéval : ainsi, la devise de Hogwarts est en latin (Draco dormiens nunquam titillandus), tout comme un certain nombre de mots de passe (par exemple, Caput draconis, formule pour entrer dans la salle commune de Gryffindor ${ }^{13}$ ) ou de sortilèges (par exemple, Expecto patronum, incantation contre les "Détraqueurs" dans Harry Potter and the Prisoner of Azkaban). Par un curieux paradoxe, d'ailleurs, la traduction latine, en intégrant ces formules au texte latin, supprime leur étrangeté ; toutefois ce phénomène est tellement limité qu’il ne contredit pas le principe général de la distanciation volontaire qui vient d'être décrit.

La principale difficulté des traductions latines d'œuvres contemporaines est liée à cet anachronisme pour ainsi dire constitutif. Comment traduire des mots qui renvoient à des réalités inconnues des anciens Romains ${ }^{14}$ ? Comment, par exemple, rendre compte en latin des objets liés au monde moderne, comme la voiture ou le train ? À moins de se lancer dans des périphrases longues et finalement incompréhensibles, il est difficile d'éviter le recours à des néologismes, à des mots qui ne sont pas attestés en latin classique. Tel est le choix de P. Needham, mais il propose très peu de créations personnelles, reprenant les traductions indiquées naguère par Carlo Egger dans son Lexicon Recentis Latinitatis 15 : " voiture » se rend donc par autocinetum, et " train " par hamaxostichus ("Hogwarts Express» se dit Hamaxostichus Rapidus Hogvartensis). Ce parti pris est cohérent, et il est probablement le meilleur : en effet, si P. Needham avait créé son propre vocabulaire, avec ses propres néologismes, il aurait dû fournir en annexe un lexique expliquant les mots qu'il venait d'inventer, ou bien le lecteur aurait dû sans cesse se reporter à l'original anglais pour comprendre les inventions du traducteur. Mais alors aurait disparu la fiction selon laquelle il pourrait exister des lecteurs connaissant seulement la traduction latine. Imaginons au contraire que de tels lecteurs existent : ils peuvent comprendre tout le vocabulaire de Harrius Potter en ayant à leur disposition un seul et unique dictionnaire, celui de C. Egger.

Les noms propres constituent des difficultés plus grandes, car il faut décider s'ils doivent être transposés ou pas. Ce problème n'est du reste pas spécifique aux traductions latines. VictorHenry Debidour ${ }^{16}$ souligne ainsi la difficulté à laquelle il fut confronté en traduisant les anthroponymes chez Aristophane : "Les deux bouts de la chaîne sont solides : il en est qu'il faut évidemment conserver, ce sont ceux des personnages historiques bien connus, Socrate, Euripide, Périclès, Cléon, etc. Il en est qu'il faut absolument "traduire". Ce sont ceux que le poète fabrique

13 Harry Potter and the Philosopher's Stone, fin du chapitre 7. La version anglaise ayant fait l'objet de multiples tirages et ces différents tirages n'étant pas tous paginés de la même façon, j'ai préféré ne pas indiquer de numéros de page. La capitulation, heureusement, ne change pas.

Voir aussi Jacqmain, "Duttilità" [n. 12], p. 6-9. Le problème se posait déjà à l'époque moderne : voir Burke, "Translations" [n. 4], p. 78-80. p. 7-17 (spéc. p. 13-14). 
en toute fantaisie et en y impliquant un sens. " Malheureusement, ajoute le traducteur, " il y a une foule de cas intermédiaires ". Dans une œuvre comme Harry Potter, on retrouve ces trois cas de figure : 1) les personnages historiques ou les lieux qui existaient déjà sous l'Antiquité ; 2) les noms propres que J.K. Rowling a créés en y incluant une signification ; 3) les " cas intermédiaires ", comme dit V.-H. Debidour, où l'on ne sait trop si les anthroponymes ou les toponymes sont motivés. Le cas (1) ne se rencontre guère que pour la ville de Londres, pour laquelle il fallait nécessairement garder le nom antique, Londinium. La frontière entre les cas (2) et (3) est en revanche assez floue. Comment décider si un nom doit être " traduit " ou pas ? Les choix de P. Needham sont extrêmement prudents : il n'a transposé que très peu de noms propres (Sprout traduit par Caulicula, Clearwater par Claraqua ${ }^{17}$ ). En revanche, il a gardé, par exemple, Malfoy (qu'il aurait pu rendre par Malefidus) ou Ravenclaw (il aurait pu essayer de traduire ce composé de " raven " et " claw" par un composé de corvus et unguis, par exemple Corvunguis). On peut critiquer cette absence de prise de risque, mais il faut reconnaître que les créations de J.K. Rowling ne sont pas toujours univoques. En outre, P. Needham est anglophone : Malfoy est transparent pour un francophone (Mal-foy $=$ « Mauvaise foi »), mais l'est sans doute moins pour un anglophone ; d'une certaine façon, les choix du traducteur sont aussi instructifs sur sa langue maternelle (l'anglais) que sur son talent de latiniste.

Quand il a dû adapter les noms propres aux déclinaisons du latin, P. Needham a choisi une solution cohérente et conforme à l'usage latin. Les Latins, lorsqu'ils déclinaient un anthroponyme étranger, grec ou hébreu par exemple, gardaient souvent le nominatif identique à la langue originale, et pour les autres cas déclinaient le nom selon le paradigme le plus proche : ainsi s'expliquent les déclinaisons du type Aeneas, -nae, Adam, -dae, ou Dauid, -idis. P. Needham a fait de même avec l'anglais, déclinant par exemple Potter, -eris ou Malfoy, -fonis. Cela donne parfois des résultats bizarres, par exemple le nom de Vous-Savez-Qui décliné sur le modèle de mors (Voldemortem, Voldemortis, etc.), sauf le nominatif qui reste Voldemort (alors qu'on aurait peutêtre attendu Voldemors) ${ }^{18}$. Certains mots s'adaptent tels quels à la troisième déclinaison, par exemple Muggles, gén. Mugglium. D'autres restent indéclinables, lorsque le cas peut être indiqué par ailleurs : dans ludus Quidditch, ce dernier mot est indéclinable, le cas étant indiqué par ludus. C'est probablement pour la même raison que le prénom du Prof. Lockhart, Gilderoy, n'est pas non plus décliné : en effet, Gilderoy n'est employé seul que comme sujet ou apostrophe, ce qui correspond en latin au nominatif et au vocatif ; comme dans tous ses autres emplois il est suivi du nom de famille Lockhart, il suffit de décliner le seul mot Lockhart (Lockhart, -artis) pour indiquer le cas du groupe nominal ${ }^{19}$.

17 Ce personnage mineur, Penelope Clearwater, apparaît à la toute fin du tome 2.

18 Petite parenthèse à propos de Vous-Savez-Qui : la traduction de You-Know-Who par Quidam est décevante, alors que Cicéron (Fam. II, 9, 1) offrait un euphémisme équivalent (Ille... scis quem dicam).

19 Par exemple, au c. 4, dans la liste des ouvrages que doivent acquérir les étudiants de deuxième année : "By Gilderoy Lockhart" est traduit par a Gilderoy Lockharte. La traduction de ce passage comporte d'ailleurs une bizarrerie : pour une raison obscure, P. Needham a modifié l'ordre des titres. Ainsi, alors qu'en anglais le premier livre à acheter est "Break with a Banshee", en latin Secessio cum Striga n'apparaît qu'en quatrième position, le dernier livre dans la version anglaise ("Year with a Yeti") devient le premier dans la version latine (Annus cum Abominandis), etc. 
Autre piège classique qui attend les traducteurs : les jeux de mots. Certes, ils ne sont pas tous rendus en latin. Par exemple, alors que le traducteur français (Jean-François Ménard) a réussi à transposer OWL, à la fois acronyme de "Ordinary Wizard Level " et nom de la chouette en anglais, par BUSE, "brevet universitaire de sorcellerie élémentaire " et oiseau assez proche de la chouette (il s'agit aussi d'un oiseau rapace), il n'en est pas de même en latin : OMG (Ordinarius Magicus Gradus) n'est pas le nom d'un oiseau et n'est même pas un nom commun. Fallait-il tenter quelque chose comme Gradus AVIS, avis restant toujours au génitif (donc inchangé dans le texte latin, seul gradus étant décliné) et signifiant "Accessus Vulgaris Incipientium Sortilegorum " ? Néanmoins, force est de reconnaitre que P. Needham a réussi à adapter plusieurs jeux de mots. Ainsi, le nom du capitaine de l'équipe de Quidditch de Gryffindor, Oliver Wood, fait l'objet d'un malentendu au chapitre 9 du premier tome : Harry Potter, entendant parler de lui par le professeur McGonagall, croit qu'il s'agit de «bois " (signification de " wood » en anglais) et s'imagine même qu'il va être puni par une cane en bois ; le double sens de Wood, rendu en français par " Dubois ", l'est aussi en latin par Silvius, anthroponyme qui est d'ailleurs attesté dans la Rome antique (selon la tradition c'est le nom du fils d'Énée, deuxième roi d'Albe) ${ }^{20}$. De même le traducteur ne pouvait éviter de transposer l'anagramme qui révèle l'identité de Riddle à fin du deuxième tome, car elle est essentielle à la compréhension du récit : TOM MARVOLO RIDDLE / I AM LORD VOLDEMORT devient TOM MUSVOX RUDDLE / SUM DUX VOLDEMORT. Cette adaptation ne rend pas compte de la polysémie de Riddle, à la fois nom propre et mot signifiant " énigme ", mais on peut s'empêcher de reconnaitre dans Musvox l'association de mus, la " souris ", et de vox, la " voix » : par son caractère loufoque, cette juxtaposition peut évoquer pour le lecteur quelque chose d'aussi étrange et "merveilleux " que Marvolo, et vox peut faire penser $\mathrm{au}$ « jeu de mots » de Riddle.

Reste enfin la grande difficulté que doit affronter tout traducteur : comment transposer les effets stylistiques? Dans le deuxième chapitre du deuxième tome, par exemple, le style officiel de la lettre du ministère de la Magie est rendu par un latin lui aussi formel. L'accent très particulier de Hagrid, en revanche, n'est pas imité : sans doute, dans le premier chapitre, son bégaiement, dû à l'émotion, «s-s-sorry » et "I c-c-can't ", est-il bien traduit par $d$-d-da mihi veniam et non p-p-possum, mais les traits originaux de sa langue, avec ses multiples élisions et déformations, ne sont pas adaptés en latin. Manifestement le traducteur n'a pas osé introduire les élisions du texte anglais, peutêtre parce qu'il a jugé qu'en latin il était malaisé d'élider les désinences qui indiquent le cas. Il était possible, pourtant, de conserver certains effets. Le latin parlé tendait à faire disparaître certaines voyelles, par exemple en position intérieure lorsqu'elles suivaient une syllabe accentuée : dans une phrase comme " this is the real myst'ry of the thing " (chapitre 4 du premier tome), traduite par rem difficilem et inexplicabilem, l'effacement du e dans " mystery " pouvait donc être rendu par les formes difficlem et inexplicablem. Et certains "vulgarismes " (par exemple des confusions entre voyelles de timbres i et e, ou de timbres u et o) auraient pu fournir des équivalents aux prononciations originales de Hagrid (" yeh » au lieu de " you », " ter » au lieu de « to », etc.).

Il est en revanche un fait stylistique que P. Needham a su remarquablement traduire : c'est l'inclusion de la poésie dans un texte en prose. Les passages rimés en anglais (dans Harrius Potter

20 Une critique toutefois à la traduction de ce passage par P. Needham : l'anglais «she was going to use " devait être traduit par uapulatura esset et non uapulaturus esset. 
et Philosophi Lapis, le chant du chapeau et celui des étudiants de Hogwarts au chapitre 7, l'énigme des bouteilles au chapitre 16) sont traduits en distiques élégiaques, ce qui suppose une maitrise remarquable de la prosodie latine.

Cet article ne prétend évidemment pas épuiser la matière : je n'ai donné que quelques exemples des problèmes posés par une traduction comme celle de Harry Potter. En outre, après avoir essayé d'analyser la traduction de P. Needham de la manière la plus sérieuse possible, je ne voudrais pas donner l'impression de me prendre trop au sérieux. Comme je l'ai déjà dit, l'une des raisons essentielles du succès d'une traduction comme Harrius Potter vient de son caractère canularesque. D'une certaine façon, cet article, qui se propose d'étudier de manière « scientifique » un texte canularesque, fait aussi partie du canular.

Jacques ELFASSI

Maître de conférences à l'Université de Metz

UFR Lettres et Langues - Ille du Saulcy

57045 Metz cedex 1

elfassi@univ-metz.fr 